\title{
Arrows, Robots, and Functional Reactive Programming
}

\author{
Paul Hudak, Antony Courtney, Henrik Nilsson, and John Peterson \\ Yale University ${ }^{\star}$ \\ Department of Computer Science \\ paul.hudak@yale.edu, antony.courtney@yale.edu, \\ henrik.nilsson@yale.edu, john.c.peterson@yale.edu
}

\begin{abstract}
Functional reactive programming, or FRP, is a paradigm for programming hybrid systems - i.e., systems containing a combination of both continuous and discrete components - in a high-level, declarative way. The key ideas in FRP are its notions of continuous, time-varying values, and time-ordered sequences of discrete events.

Yampa is an instantiation of FRP as a domain-specific language embedded in Haskell. This paper describes Yampa in detail, and shows how it can be used to program a particular kind of hybrid system: a mobile robot. Because performance is critical in robotic programming, Yampa uses arrows (a generalization of monads) to create a disciplined style of programming with time-varying values that helps ensure that common kinds of time- and space-leaks do not occur.

No previous experience with robots is expected of the reader, although a basic understanding of physics and calculus is assumed. No knowledge of arrows is required either, although we assume a good working knowledge of Haskell.

This paper is dedicated in memory of Edsger W. Dijkstra

for his influential insight that mathematical logic is and must be the basis for sensible computer program construction.
\end{abstract}

\section{Introduction}

Can functional languages be used in the real world, and in particular for realtime systems? More specifically, can the expressiveness of functional languages be used advantageously in such systems, and can performance issues be overcome at least for the most common applications?

For the past several years we have been trying to answer these questions in the affirmative. We have developed a general paradigm called functional reactive

\footnotetext{
* This research was supported in part by grants from the National Science Foundation (CCR9900957 and CCR-9706747), the Defense Advanced Research Projects Agency (F33615-99-C-3013 and DABT63-00-1-0002), and the National Aeronautics and Space Administration (NCC 2-1229). The second author was also supported by an NSF Graduate Research Fellowship.
} 
programming that is well suited to programming hybrid systems, i.e. systems with both continuous and discrete components. An excellent example of a hybrid system is a mobile robot. From a physical perspective, mobile robots have continuous components such as voltage-controlled motors, batteries, and range finders, as well as discrete components such as microprocessors, bumper switches, and digital communication. More importantly, from a logical perspective, mobile robots have continuous notions such as wheel speed, orientation, and distance from a wall, as well as discrete notions such as running into another object, receiving a message, or achieving a goal.

Functional reactive programming was first manifested in Fran, a domain specific language (DSL) for graphics and animation developed by Conal Elliott at Microsoft Research [5,4]. FRP $[13,8,16]$ is a DSL developed at Yale that is the "essence" of Fran in that it exposes the key concepts without bias toward application specifics. FAL [6], Frob [11,12], Fvision [14], and Fruit [2] are four other DSLs that we have developed, each embracing the paradigm in ways suited to a particular application domain. In addition, we have pushed FRP toward real-time embedded systems through several variants including Real-Time FRP and Event-Driven FRP $[18,17,15]$.

The core ideas of functional reactive programming have evolved (often in subtle ways) through these many language designs, culminating in what we now call Yampa, which is the main topic of this paper. ${ }^{1}$ Yampa is a DSL embedded in Haskell and is a refinement of FRP. Its most distinguishing feature is that the core FRP concepts are represented using arrows [7], a generalization of monads. The programming discipline induced by arrows prevents certain kinds of timeand space-leaks that are common in generic FRP programs, thus making Yampa more suitable for systems having real-time constraints.

Yampa has been used to program real industrial-strength mobile robots [10, $8]^{2}$, building on earlier experience with FRP and Frob $[11,12]$. In this paper, however, we will use a robot simulator. In this way, the reader will be able to run all of our programs, as well as new ones that she might write, without having to buy a $\$ 10,000$ robot. All of the code in this paper, and the simulator itself, are available via the Yampa home page at www.haskell.org/yampa.

The simulated robot, which we refer to as a simbot, is a differential drive robot, meaning that it has two wheels, much like a cart, each driven by an independent motor. The relative velocity of these two wheels thus governs the turning rate of the simbot; if the velocities are identical, the simbot will go straight. The physical simulation of the simbot includes translational inertia, but (for simplicity) not rotational inertia.

The motors are what makes the simbot go; but it also has several kinds of sensors. First, it has a bumper switch to detect when the simbot gets "stuck."

\footnotetext{
${ }^{1}$ Yampa is a river in Colorado whose long placid sections are occasionally interrupted by turbulent rapids, and is thus a good metaphor for the continuous and discrete components of hybrid systems. But if you prefer acronyms, Yampa was started at YAle, ended in Arrows, and had Much Programming in between.

${ }^{2}$ In these two earlier papers we referred to Yampa as AFRP.
} 
That is, if the simbot runs into something, it will just stop and signal the program. Second, it has a range finder that can determine the nearest object in any given direction. In our examples we will assume that the simbot has independent range finders that only look forward, backward, left, and right, and thus we will only query the range finder at these four angles. Third, the simbot has what we call an "animate object tracker" that gives the location of all other simbots, as well as possibly some free-moving balls, that are within a certain distance from the simbot. You can think of this tracker as modelling either a visual subsystem that can see these objects, or a communication subsystem through which the simbots and balls share each others' coordinates. Each simbot also has a unique ID and a few other capabilities that we will introduce as we need them.

\section{Yampa Basics}

The most important concept underlying functional reactive programming is that of a signal: a continous, time-varying value. One can think of a signal as having polymorphic type:

$$
\text { Signal a }=\text { Time } \rightarrow \text { a }
$$

That is, a value of type Signal a is a function mapping suitable values of time (Double is used in our implementation) to a value of type a. Conceptually, then, a signal $s$ 's value at some time $t$ is just $s(t)$.

For example, the velocity of a differential drive robot is a pair of numbers representing the speeds of the left and right wheels. If the speeds are in turn represented as type Speed, then the robot's velocity can be represented as type Signal (Speed, Speed). A program controlling the robot must therefore provide such a value as output.

Being able to define and manipulate continuous values in a programming language provides great expressive power. For example, the equations governing the motion of a differential drive robot [3] are:

$$
\begin{aligned}
& x(t)=\frac{1}{2} \int_{0}^{t}\left(v_{r}(t)+v_{l}(t)\right) \cos (\theta(t)) d t \\
& y(t)=\frac{1}{2} \int_{0}^{t}\left(v_{r}(t)+v_{l}(t)\right) \sin (\theta(t)) d t \\
& \theta(t)=\frac{1}{l} \int_{0}^{t}\left(v_{r}(t)-v_{l}(t)\right) d t
\end{aligned}
$$

where $x(t), y(t)$, and $\theta(t)$ are the robot's $x$ and $y$ coordinates and orientation, respectively; $v_{r}(t)$ and $v_{l}(t)$ are the right and left wheel speeds, respectively; and $l$ is the distance between the two wheels. In FRP these equations can be written as:

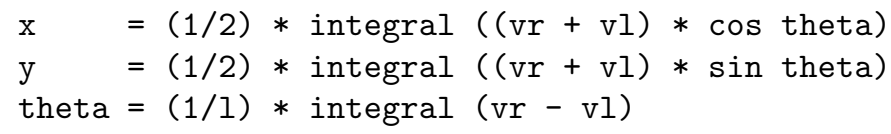


All of the values in this FRP program are implicitly time-varying, and thus the explicit time $t$ is not present. ${ }^{3}$ Nevertheless, the direct correspondence between the physical equations (i.e. the specification) and the FRP code (i.e. the implementation) is very strong.

\subsection{Arrowized FRP}

Although quite general, the concept of a signal can lead to programs that have conspicuous time- and space-leaks, ${ }^{4}$ for reasons that are beyond the scope of this paper. Earlier versions of Fran, FAL, and FRP used various methods to make this performance problem less of an issue, but ultimately they all either suffered from the problem in one way or another, or introduced other problems as a result of fixing it.

In Yampa, the problem is solved in a more radical way: signals are simply not allowed as first-class values! Instead, the programmer has access only to signal transformers, or what we prefer to call signal functions. A signal function is just a function that maps signals to signals:

$$
\text { SF a b = Signal a } \rightarrow \text { Signal } b
$$

However, the actual representation of the type SF in Yampa is hidden (i.e. SF is abstract), so one cannot directly build signal functions or apply them to signals. Instead of allowing the user to define arbitrary signal functions from scratch (which makes it all too easy to introduce time- and space-leaks), we provide a set of primitive signal functions and a set of special composition operators (or "combinators") with which more complex signal functions may be defined. Together, these primitive values and combinators provide a disciplined way to define signal functions that, fortuitously, avoids time- and space-leaks. We achieve this by structuring Yampa based on arrows, a generalization of monads proposed in [7]. Specifically, the type SF is made an instance of the Arrow class.

So broadly speaking, a Yampa program expresses the composition of a possibly large number of signal functions into a composite signal function that is then "run" at the top level by a suitable interpreter. A good analogy for this idea is a state or IO monad, where the state is hidden, and a program consists of a linear sequencing of actions that are eventually run by an interpreter or the operating system. But in fact arrows are more general than monads, and in particular the composition of signal functions does not have to be completely linear, as will be illustrated shortly. Indeed, because signal functions are abstract, we should

\footnotetext{
${ }^{3}$ This implies that the sine, cosine, and arithmetic operators are over-loaded to handle signals properly.

4 A time-leak in a real-time system occurs whenever a time-dependent computation falls behind the current time because its value or effect is not needed yet, but then requires "catching up" at a later point in time. This catching up process can take an arbitrarily long time, and may or may not consume space as well. It can destroy any hope for real-time behavior if not managed properly.
} 
be concerned that we have a sufficient set of combinators to compose our signal functions without loss of expressive power.

We will motivate the set of combinators used to compose signal functions by using an analogy to so-called "point-free" functional programming (an example of which is the Bird/Meertens formalism [1]). For the simplest possible example, suppose that $f 1:: A \rightarrow B$ and $f 2:: B \rightarrow C$. Then instead of writing:

$$
\begin{aligned}
& g:: A \rightarrow C \\
& g x=f 2(f 1 x)
\end{aligned}
$$

we can write in a point-free style using the familiar function composition operator:

$$
g=f 2 \cdot f 1
$$

This code is "point-free" in that the values (points) passed to and returned from a function are never directly manipulated.

To do this at the level of signal functions, all we need is a primitive operator to "lift" ordinary functions to the level of signal functions:

$$
\operatorname{arr}::(\mathrm{a} \rightarrow \mathrm{b}) \rightarrow \mathrm{SF} \text { a b }
$$

and a primitive combinator to compose signal functions:

$$
(\gg>):: \mathrm{SF} \text { a } \mathrm{b} \rightarrow \mathrm{SF} \text { b } \mathrm{c} \rightarrow \mathrm{SF} \text { a } \mathrm{c}
$$

We can then write:

$$
\begin{aligned}
g^{\prime} & : \text { : SF A C } \\
g^{\prime} & =\operatorname{arr} g \\
& =\operatorname{arr} f 1 \gg \text { arr } f 2
\end{aligned}
$$

Note that (>>>) actually represents reverse function composition, and thus its arguments are reversed in comparison to (.).

Unfortunately, most programs are not simply linear compositions of functions, and it is often the case that more than one input and/or output is needed. For example, suppose that $\mathrm{f} 1:: \mathrm{A} \rightarrow \mathrm{B}, \mathrm{f} 2:: \mathrm{A} \rightarrow \mathrm{C}$ and we wish to define the following in point-free style:

$$
\begin{aligned}
& \mathrm{h}:: \mathrm{A} \rightarrow(B, C) \\
& \mathrm{h} x=(\mathrm{f} 1 \mathrm{x}, \mathrm{f} 2 \mathrm{x})
\end{aligned}
$$

Perhaps the simplest way is to define a combinator:

$$
\begin{aligned}
& (\&)::(a->b) \rightarrow(a->c) \rightarrow a \rightarrow(b, c) \\
& (f 1 \& f 2) x=(f 1 x, f 2 x)
\end{aligned}
$$

which allows us to define $\mathrm{h}$ simply as:

$\mathrm{h}=\mathrm{f} 1 \& \mathrm{f} 2$ 
In Yampa there is a combinator (\&\&\&) : : SF a b $\rightarrow \mathrm{SF}$ a $\mathrm{c} \rightarrow \mathrm{SF}$ a $(\mathrm{b}, \mathrm{c})$ that is analogous to \&, thus allowing us to write:

$$
\begin{aligned}
h^{\prime} & :: \operatorname{SF} A(B, C) \\
h^{\prime} & =\operatorname{arr} h \\
& =\operatorname{arr} f 1 \text { \&\&\& arr } f 2
\end{aligned}
$$

As another example, suppose that $f 1:: A \rightarrow B$ and $f 2:: C \rightarrow$ D. One can easily write a point-free version of:

$$
\begin{aligned}
& i::(A, C)->(B, D) \\
& i(x, y)=(f 1 x, f 2 y)
\end{aligned}
$$

by using (\&) defined above and Haskell's standard fst and snd operators:

$$
i=(f 1 \text {. fst) \& (f2. snd) }
$$

For signal functions, all we need are analogous versions of $\mathrm{f}$ st and snd, which we can achieve via lifting:

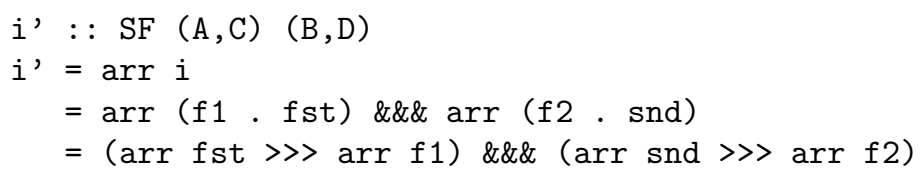

The "argument wiring" pattern captured by $i$ ' is in fact a common one, and thus Yampa provides the following combinator:

$(* * *):: \mathrm{SF} b \mathrm{c}^{\prime} \rightarrow \mathrm{SF} \mathrm{b}^{\prime} \mathrm{c}^{\prime} \rightarrow \mathrm{SF}\left(\mathrm{b}, \mathrm{b}^{\prime}\right)\left(\mathrm{c}, \mathrm{c}^{\prime}\right)$

$f * * * g=(\operatorname{arr} f s t>>f) \& \& \&(\operatorname{arr}$ snd $\gg>g$ )

so that $i$ ' can be written simply as:

$i^{\prime}=\operatorname{arr} \mathrm{f} 1 * * * \operatorname{arr} \mathrm{f} 2$

$g^{\prime}, h^{\prime}$, and i' were derived from $g, h$, and $i$, respectively, by appealing to one's intuition about functions and their composition. In the next section we will formalize this using type classes.

\subsection{The Arrow Class}

One could go on and on in this manner, adding combinators as they are needed to solve particular "argument wiring" problems, but it behooves us at some point to ask if there is a minimal universal set of combinators that is sufficient to express all possible wirings. Note that so far we have introduced three combinators arr, ( >>), and (\&\&\&) - without definitions, and a fourth - (***) - was defined in terms of these three. Indeed these three combinators constitute a minimal universal set.

However, this is not the only minimal set. In fact, in defining the original Arrow class, Hughes instead chose the set arr, ( $>>)$, and first: 


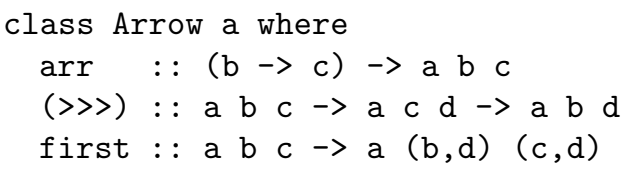

where first is analogous to the following function defined at the ordinary function level:

firstfun $f=\backslash(x, y) \rightarrow(f x, y)$

In Yampa, the type SF is an instance of class Arrow, and thus these types are consistent with what we presented earlier. To help see how this set is miminal, here are definitions of second and (\&\&\&) in terms of the Arrow class methods:

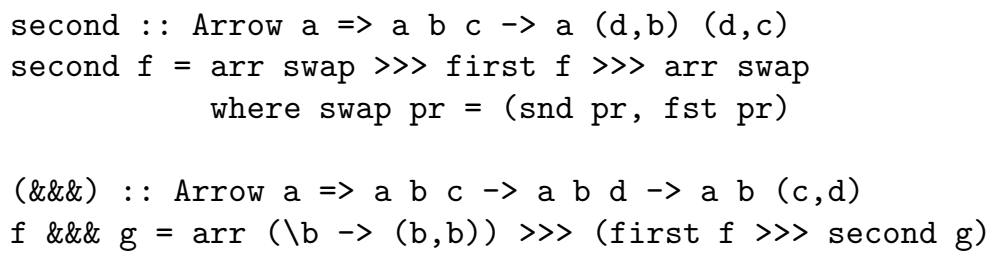

In addition, here is an instance declaration that shows how Haskell's normal function type can be treated as an arrow:

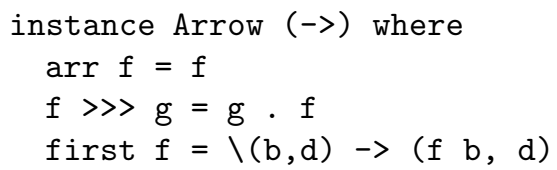

With this instance declaration, the derivations of $g^{\prime}, h^{\prime}$, and $i^{\prime}$ in the previous section can be formally justified.

Exercise 1. Define (a) first in terms of just arr, (>>>), and (\&\&\&), (b) (***) in terms of just first, second, and ( $\gg>)$, and (c) (\&\&\&) in terms of just arr, $(\gg \gg)$, and $(* * *)$.

\subsection{Commonly Used Combinators}

In practice, it is better to think in terms of a commonly-used set of combinators rather than a minimal set. Figure 1 shows a set of eight combinators that we use often in Yampa programming, along with the graphical "wiring of arguments" that five of them imply.

Yampa also provides many convenient library functions that facilitate programming in the arrow framework. Here are some that we will use later in this paper:

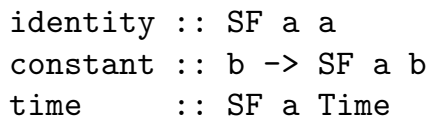




\begin{tabular}{|c|c|}
\hline $\operatorname{arr}$ & $::$ Arrow $a \Rightarrow(b \rightarrow c) \rightarrow a b c$ \\
\hline$(\gg>>)$ & $::$ Arrow $a \Rightarrow a b c \rightarrow a c d \rightarrow a b d$ \\
\hline$(<<<)$ & $:$ Arrow $a \Rightarrow a c d \rightarrow a b c \rightarrow a b d$ \\
\hline first & $:$ Arrow $a \Rightarrow a b a->a(b, d)(c, d)$ \\
\hline second & $:$ Arrow $a \Rightarrow a b c \rightarrow a(d, b)(d, c)$ \\
\hline$(* * *)$ & $:$ Arrow $a \Rightarrow a b c \rightarrow a b^{\prime} c^{\prime} \rightarrow a\left(b, b^{\prime}\right)\left(c, c^{\prime}\right)$ \\
\hline$(\& \& \&)$ & $:$ Arrow $a \Rightarrow a b c \rightarrow a b c^{\prime} \rightarrow a b\left(c, c^{\prime}\right)$ \\
\hline loop & $:$ Arrow $a \Rightarrow a(b, d)(c, d) \rightarrow a b c$ \\
\hline
\end{tabular}

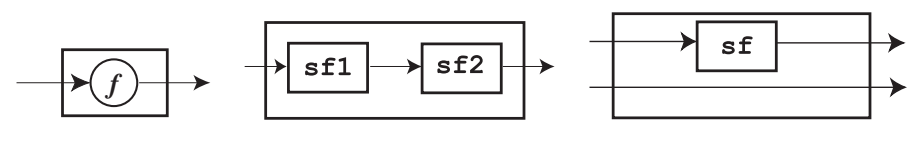
(a) $\operatorname{arr} f$
(b) sf1 $\gg>$ sf2
(c) first sf

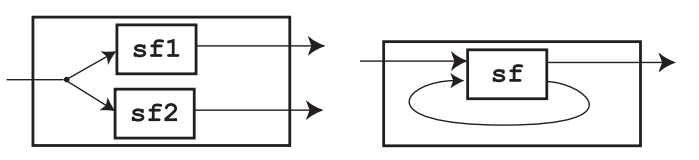
(d) sf1 \&\&\& sf2
(e) loop sf

Fig. 1. Commonly Used Arrow Combinators 
The identity signal function is analogous to the identity function in Haskell, and in fact is equivalent to arr id. The constant function is useful for generating constant signal functions, is analogous to Haskell's const function, and in fact is equivalent to arr . const. Finally, time is a signal function that yields the current time, and is equivalent to constant $1.0 \gg$ integral, where integral is a pre-defined Yampa signal function with type: ${ }^{5}$

integral :: SF Double Double

Yampa also defines a derivative signal function.

It is important to note that some signal functions are stateful, in that they accumulate information over time. integral is a perfect example of such a function: by definition, it sums instantaneuous values of a signal over time. Stateful signal functions cannot be defined using arr, which only lifts pure functions to the level of arrows. Stateful functions must either be pre-defined or be defined in terms of other stateful signal functions.

Stated another way, stateful signal functions such as integration and differentiation depend intimately on the underlying time-varying semantics, and so do not have analogous unlifted forms. Indeed, it is so easy to lift unary functions to the level of signal functions that there is generally no need to provide special signal function versions of them. For example, instead of defining a special sinSF, cosSF, etc., we can just use arr sin, arr cos, etc. Furthermore, with the binary lifting operator:

$\operatorname{arr} 2::(a->b->c) \rightarrow S F(a, b) c$

$\operatorname{arr} 2=\operatorname{arr}$. uncurry

we can also lift binary operators. For example, arr2 (+) has type Num a $\Rightarrow$ SF $(a, a)$ a.

\subsection{A Simple Example}

To see all of this in action, consider the FRP code presented earlier for the coordinates and orientation of the mobile robot. We will rewrite the code for the $\mathrm{x}$-coordinate in Yampa (leaving the y-coordinate and orientation as an exercise).

Suppose there are signal functions vrSF, vISF : : SF SimbotInput Speed and thetaSF : : SF SimbotInput Angle. The type SimbotInput represents the input state of the simbot, which we will have much more to say about later. With these signal functions in hand, the previous FRP code for $\mathrm{x}$ :

$$
\mathrm{x}=(1 / 2) * \text { integral }((\mathrm{vr}+\mathrm{vl}) * \cos \text { theta })
$$

can be rewritten in Yampa as:

5 This function is actually overloaded for any vector space, but that does not concern us here, and thus we have specialized it to Double. 


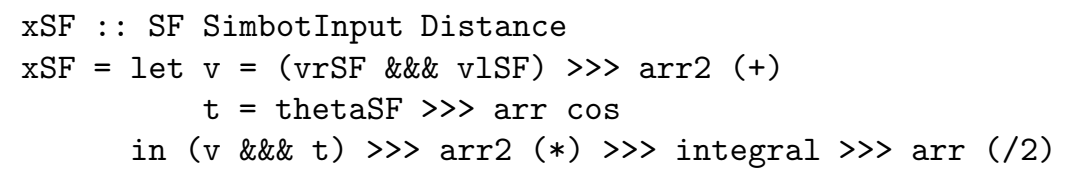

Exercise 2. Define signal functions ySF and thetaSF in Yampa that correspond to the definitions of $y$ and theta, respectively, in FRP.

\subsection{Arrow Syntax}

Although we have achieved the goal of preventing direct access to signals, one might argue that we have lost the clarity of the original FRP code: the code for xSF is certainly more difficult to understand than that for $\mathrm{x}$. Most of the complexity is due to the need to wire signal functions together using the various pairing/unpairing combinators such as (\&\&\&) and (***). Precisely to address this problem, Paterson [9] has suggested the use of special syntax to make arrow programming more readable, and has written a preprocessor that converts the syntactic sugar into conventional Haskell code. Using this special arrow syntax, the above Yampa code for xSF can be rewritten as:

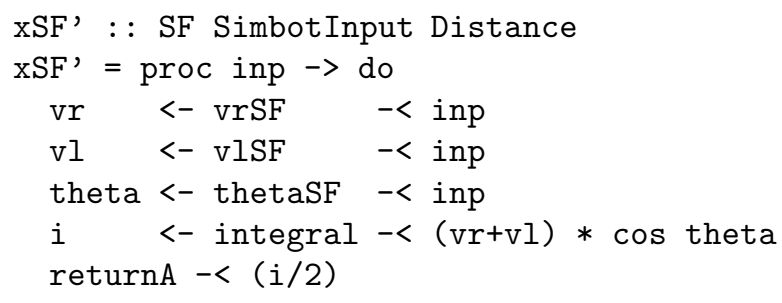

Although not quite as readable as the original FRP definition of $\mathrm{x}$, this code is far better than the unsugared version. There are several things to note about the structure of this code:

1. The syntax proc pat $\rightarrow \ldots$ is analogous to a Haskell lambda expression of the form \pat $\rightarrow \ldots$, except that it defines a signal function rather than a normal Haskell function.

2. In the syntax pat $<-S F e x p r-<$ expr, the expression SFexpr must be a signal function, say of type SF T1 T2, in which case expr must have type $\mathrm{T} 1$ and pat must have type T2. This is analogous to pat $=\operatorname{expr}_{1} \operatorname{expr}_{2}$ in a Haskell let or where clause, in which case if $\operatorname{expr}_{1}$ has type T1 $\rightarrow \mathrm{T} 2$, then expr $_{2}$ must have type T1 and pat must have type T2.

3. The overall syntax:

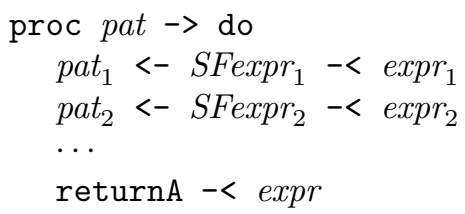


defines a signal function. If pat has type T1 and expr has type T2, then the type of the signal function is SF T1 T2. In addition, any variable bound by one of the patterns pat $_{i}$ can only be used in the expression expr or in an expression $\operatorname{expr}_{j}$ where $j>i$. In particular, it cannot be used in any of the signal function expressions SFexpr $_{i}$.

It is important to note that the arrow syntax allows one to get a handle on a signal's values (or samples), but not on the signals themselves. In other words, first recalling that a signal function SF a b can be thought of as a type Signal a $\rightarrow$ Signal b, which in turn can be thought of as type

(Time $\rightarrow$ a) $\rightarrow$ (Time $\rightarrow$ b), the syntax allows getting a handle on values of type $a$ and $b$, but not on values of type Time $\rightarrow$ a or Time $\rightarrow$ b.

Figure 2(a) is a signal flow diagram that precisely represents the wiring implied by the sugared definition of xSF'. (It also reflects well the data dependencies in the original FRP program for $\mathrm{x}$.) Figure 2(b) shows the same diagram, except that it has been overlaid with the combinator applications implied by the unsugared definition of $\mathrm{xSF}$ (for clarity, the lifting via arr of the primitive functions - i.e. those drawn with circles - is omitted). These diagrams demonstrate nicely the relationship between the sugared and unsugared forms of Yampa programs.

Exercise 3. Rewrite the definitions of ySF and thetaSF from the previous exercise using the arrow syntax. Also draw their signal flow diagrams.

\subsection{Discrete Events and Switching}

Most programming languages have some kind of conditional choice capability, and Yampa is no exception. Indeed, given signal functions flag : SF a Bool and sfx, sfy : : SF a b, then the signal function:

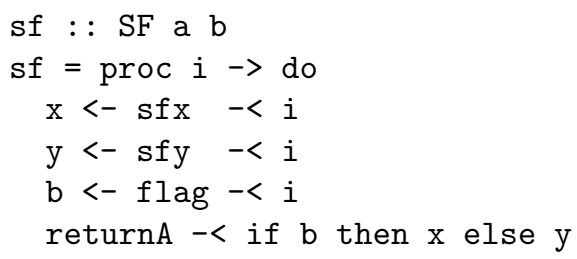

behaves like sfx whenever flag yields a true value, and like sfy whenever it yields false.

However, this is not completely satisfactory, because there are many situations where one would prefer that a signal function switch into, or literally become, some other signal function, rather than continually alternate between two signal functions based on the value of a boolean. Indeed, there is often a succession of new signal functions to switch into as a succession of particular events occurs, much like state changes in a finite state automaton. Furthermore, we would like for these newly invoked signal functions to start afresh from time zero, rather than being signal functions that have been "running" since the 


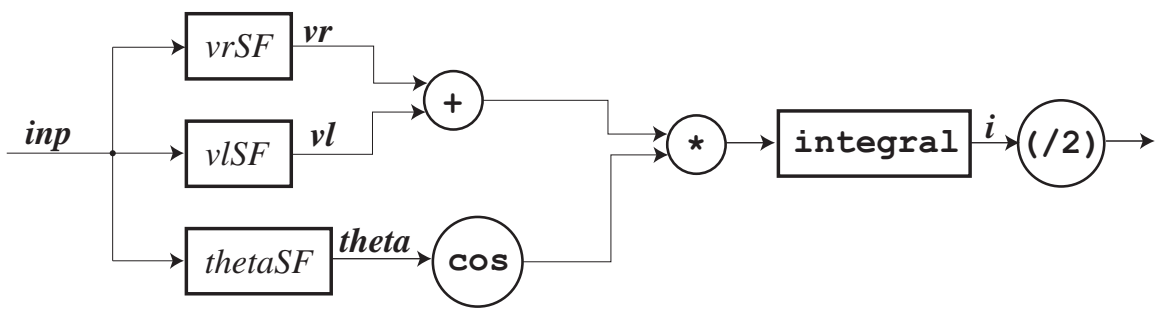

(a) Sugared

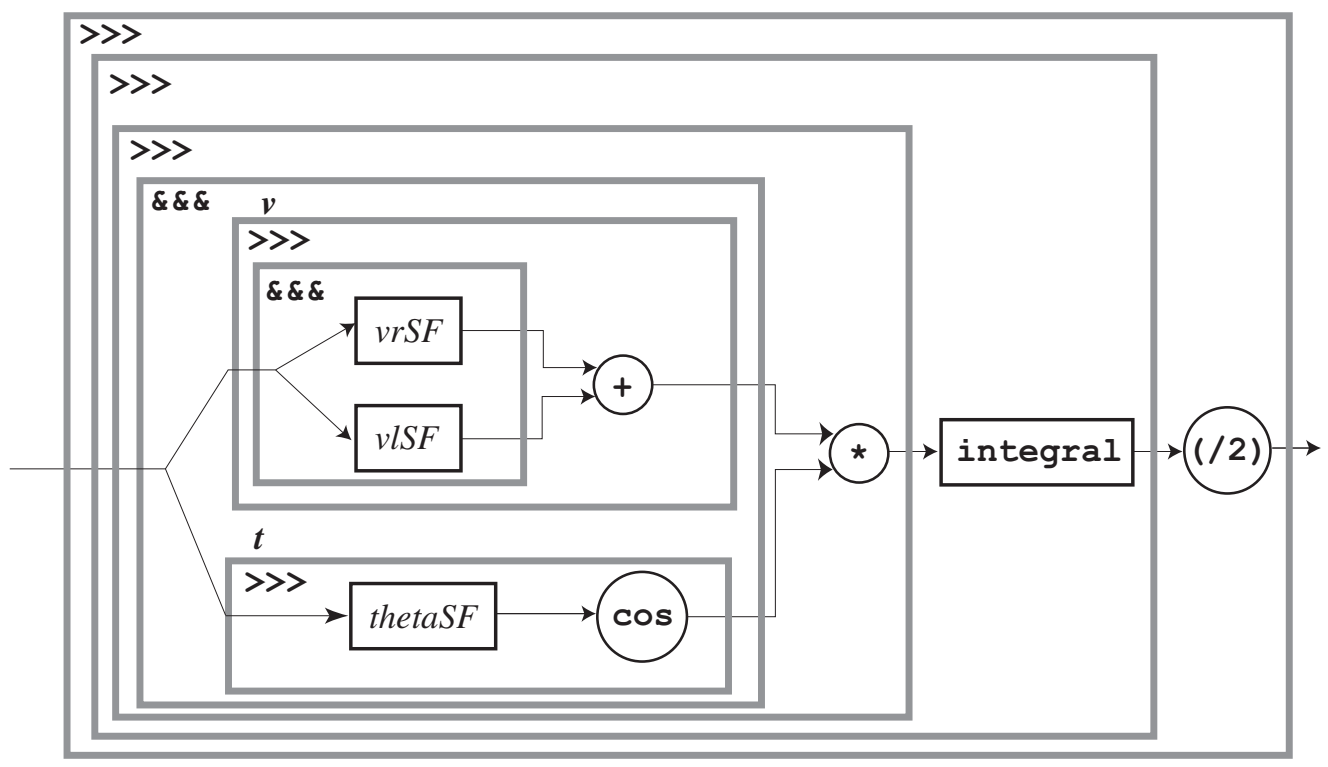

(b) Unsugared

Fig. 2. Signal Flow Diagrams for xSF 
program began. This relates precisely to the issue of "statefulness" that was previously discussed.

This advanced functionality is achieved in Yampa using events and switching combinators.

In previous versions of FRP, including Fran, Frob, and FAL, a significant distinction was made between continuous values and discrete events. In Yampa this distinction is not as great. Events in Yampa are just abstract values that are isomorphic to Haskell's Maybe data type. A signal of type Signal (Event b) is called an event stream, and is a signal that, at any point in time, yields either nothing or an event carrying a value of type b. A signal function of type $\mathrm{SF}$ a (Event b) generates an event stream, and is called an event source.

Note: Although event streams and continuous values are both represented as signals in Yampa, there are important semantic differences between them. For example, improper use of events may lead to programs that are not convergent, or that allow the underlying sampling rate to "show through" in the program's behavior. Semantically speaking, event streams in Yampa should not be "infinitely dense" in time; practically speaking, their frequency should not exceed the internal sampling rate unless buffering is provided. ${ }^{6}$

As an example of a well-defined event source, the signal function:

rsStuck :: SF SimbotInput (Event ())

generates an event stream whose events correspond to the moments when the robot gets "stuck:" that is, an event is generated every time the robot's motion is blocked by an obstacle that it has run into.

What makes event streams special is that there is a special set of functions that use event streams to achieve various kinds of switching. The simplest switching combinator is called switch, whose type is given by:

switch : : SF a (b, Event $c) \rightarrow(c \rightarrow S F$ a b) $\rightarrow$ SF a b

The expression (sf1 \&\&\& es) 'switch' $\backslash e->$ sf2 behaves as sf1 until the first event in the event stream es occurs, at which point the event's value is bound to e and the behavior switches over to sf 2 .

For example, in order to prevent damage to a robot wheel's motor, we may wish to set its speed to zero when the robot gets stuck:

xspd : : Speed $\rightarrow$ SF SimbotInput Speed

xspd $\mathrm{v}=$ (constant $\mathrm{v} \& \& \&$ rsStuck) 'switch' $\backslash() \rightarrow$ constant 0

It should be clear that stateful Yampa programs can be constructed using switching combinators.

Exercise 4. Rather than set the wheel speed to zero when the robot gets stuck, negate it instead. Then define xspd recursively so that the velocity gets negated every time the robot gets stuck.

${ }^{6}$ Certain input events such as key presses are in fact properly bufferred in our implementation such that none will be lost. 
Switching semantics. There are several kinds of switching combinators in Yampa, four of which we will use in this paper. These four switchers arise out of two choices in the semantics:

1. Whether or not the switch happens exactly at the time of the event, or infinitesimally just after. In the latter case, a "d" (for "delayed") is prefixed to the name switch.

2. Whether or not the switch happens just for the first event in an event stream, or for every event. In the latter case, an "r" (for "recurring") is prefixed to the name switch.

This leads to the four switchers, whose names and types are:

switch, dSwitch : : SF a (b, Event c) $\rightarrow$ (c $\rightarrow$ SF a b) $\rightarrow$ SF a b rSwitch, drSwitch : : SF a b $\rightarrow$ SF ( $a$, Event (SF a b)) b

An example of the use of switch was given above. Delayed switching is useful for certain kinds of recursive signal functions. In Sec. 2.7 we will see an example of the use of drSwitch.

As mentioned earlier, an important property of switching is that time begins afresh within each signal function being switched into. For example, consider the expression:

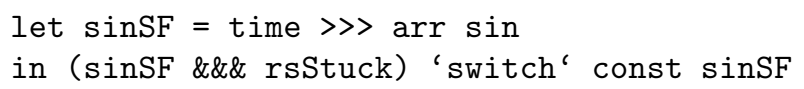

sinSF to the left of the switch generates a sinusoidal signal. If the first event generated by rsStuck happens at time $t$, then the sinSF on the right will begin at time 0 , regardless of what the time $t$ is; i.e. the sinusoidal signal will start over at the time of the event.

Useful event functions. Event is an instance of class Functor, and thus fmap can be used to change the value carried by an event. For example, we can increment the value of an event e : : Event Double by writing fmap (+1) e. Sometimes we don't care about the old value of an event when creating a new one, so Yampa also provides:

tag : : Event $a \rightarrow b \rightarrow$ Event $b$

e 'tag' b = fmap (const b) e

It is often desirable to merge events; for example, to form the disjunction of two logical events. The only problem is deciding what to do with simultaneous events. The most general form of merge:

mergeBy : : (a $\rightarrow$ a $\rightarrow$ a) $\rightarrow$ Event a $\rightarrow$ Event a $\rightarrow$ Event a

allows the user to decide how to handle simultaneous events by providing a function to combine the event values. Alternatively, one may choose to give preference to the left or right event: 


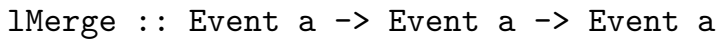

If there is no possibility of simultaneous events, merge may be used, which generates an error if in fact two events occur together:

merge : : Event a $\rightarrow$ Event a $\rightarrow$ Event a

So far we have only considered pre-existing events. Some of these may come from external sources, such as a bumper switch or communications subsystem, but it is often convenient to define our own events. Yampa provides a variety of ways to generate new events, the most important being:

edge : : SF Bool (Event ())

The expression boolSF $\gg>$ edge generates an event every time the signal from boolSF goes from False to True (i.e. the "leading edge" of the signal). For example, if tempSF : : SF SimbotInput Temp is a signal function that indicates temperature, then:

alarmSF :: SF SimbotInput (Event ())

$\operatorname{alarmSF}=$ tempSF $\gg$ arr $(>100) \gg>$ edge

generates an alarm event if the temperature exceeds 100 degrees.

Here are a few other useful event generation functions:

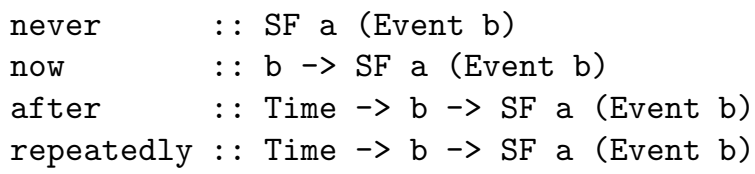

never is an event source that never generates any event occurrences. now $v$ generates exactly one event, whose time of occurrence is zero (i.e. now) and whose value is $\mathrm{v}$. The expression after $\mathrm{t} \mathrm{v}$ generates exactly one event, whose time of occurrence is $t$ and whose value is $v$. Similarly, repeatedly $t v$ generates an event every $t$ seconds, each with value $\mathrm{v}$.

To close this section, we point out that the discrete and continuous worlds interact in important ways, with switching, of course, being the most fundamental. But Yampa also provides several other useful functions to capture this interaction. Here are two of them:

hold : : a $\rightarrow$ SF (Event a) a

accum : : a $\rightarrow$ SF (Event $(a->a))$ (Event a)

The signal function hold $\mathrm{v}$ initially generates a signal with constant value $\mathrm{v}$, but every time an event occurs with value v', the signal takes on (i.e. "holds") that new value v'. The signal function accum v0 is assentially an event stream transformer. Each input event generates one output event. If $f_{n}$ is the function corresponding to the $n$th input event, then the value $v_{n}$ of the $n$th output event is just $f_{n} v_{n-1}$, for $n \geq 1$, and with $v_{0}=\mathrm{v} 0$.

For example, the following signal function represents the number of alarms generated from alarmSF defined earlier: 
alarmCountSF : : SF SimbotInput Int

alarmCountSF = alarmSF $\gg$ arr ('tag' (+1)) $\gg>$ accum $0 \gg>$ hold 0

Indeed, the accum followed by hold idiom is so common that it is predefined in Yampa:

accumHold : : a $\rightarrow$ SF (Event $(a->$ a)) a

accumHold init $=$ accum init $\gg$ hold init

Exercise 5. Suppose v :: SF SimbotInput Velocity represents the scalar velocity of a simbot. If we integrate this velocity, we get a measure of how far the simbot has traveled. Define an alarm that generates an event when either the simbot has traveled more than d meters, or it has gotten stuck.

\subsection{Recursive Signals}

Note in Fig. 1 the presence of the loop combinator. Its purpose is to define recursive signal functions; i.e. it is a fixpoint operator. The arrow syntax goes one step further by allowing recursive definitions to be programmed directly, which the preprocessor expands into applications of the loop combinator. In this case the user must include the keyword rec prior to the collection of recursive bindings.

For example, a common need when switching is to take a "snapshot" of the signal being switched out of, for use in computing the value of the signal being switched into. Suppose that there is an event source

incrVelEvs : : SF SimbotInput (Event ()) whose events correspond to commands to increment the velocity. We can define a signal function that responds to these commands as follows:

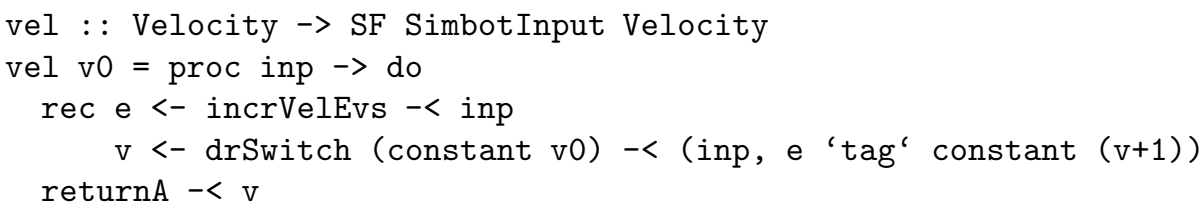

Note that $\mathrm{v}$ is recursively defined. This requires the use of the rec keyword, and also the use of a delayed switch to ensure that the recursion is well founded. Also note that the recurring version of switch is used, because we want the velocity update to happen on every event. Finally, note the use of tag to update the value of an event.

The need for a delayed switch is perhaps best motivated by analogy to recursively defined lists, or streams. The definition:

ones $=1$ : ones

expresses the usual infinite stream of ones, and is obviously well founded, whereas the list:

ones $=$ ones 
is obviously not well founded. The value of 1 placed at the front of the list can be thought of as a delay in the access of ones. That is the idea behind a delayed switch, although semantically the delay is intended to be infinitesimally small, and in the implementation we avoid introducing a delay that could affect performance.

Exercise 6. Redefine vel using dSwitch instead of drSwitch, and without using the rec keyword. (Hint: define vel recursively instead of defining v recursively.)

\section{Programming the Robot Simulator}

\subsection{Robot Input and Output}

Generally speaking, one might have dozens of different robots, some real, some simulated, and each with different kinds of functionality (two wheels, three wheels, four wheels, cameras, sonars, bumper switches, actuators, speakers, flashing lights, missle launchers, and so on). These differences are captured in the input and output types of the robot. For example, there is only one kind of simulated robot, or simbot, whose input type is SimbotInput and whose output type is SimbotOutput.

[Note: The code described in this section works with Yampa version 0.9 (and 0.9.x patches), but some changes are anticipated for use with future Yampa versions 1.0 and higher. In particular, the module names will change. In Yampa 0.9 they are still known under their old names (AFrob, AFrobRobotSim, etc.) for backwards compatibility reasons.]

We refer to the collection of Yampa libraries that are robot-specific as AFrob. The AFrob library was written to be as generic as possible, and thus it does not depend directly on the robot input and output types. Rather, type classes are used to capture different kinds of functionality. Each robot type is an instance of some subset of these classes, depending on the functionality it has to offer.

For example, SimbotInput is a member of the type classes shown in the upper half of Fig. 3, and SimbotOutput is a member of the lower ones. The types Velocity, Distance, Angle, RotVel, RotAcc, Length, Acceleration, Speed, Heading, and Bearing are all synonyms for type Double. Type Position2 is a synonym for Point2 Position, where:

data Realfloat a $\Rightarrow$ Point2 a = Point2 !a !a

deriving Eq

We will give examples of the use of many of these operations and type classes in the examples that follow. Before doing so, however, there is one other detail to describe about the output classes. Note in Fig. 3 that the methods in the last two classes return a type MR a, where a is constrained to be a MergeableRecord. This allows one to incrementally specify certain "fields" of the record, and to merge them later. There are two key operations on mergeable records:

mrMerge : : MergeableRecord a $\Rightarrow$ MR a $\rightarrow$ MR a $\rightarrow$ MR a

mrFinalize : : MergeableRecord $a \Rightarrow M R$ a $\rightarrow$ a 


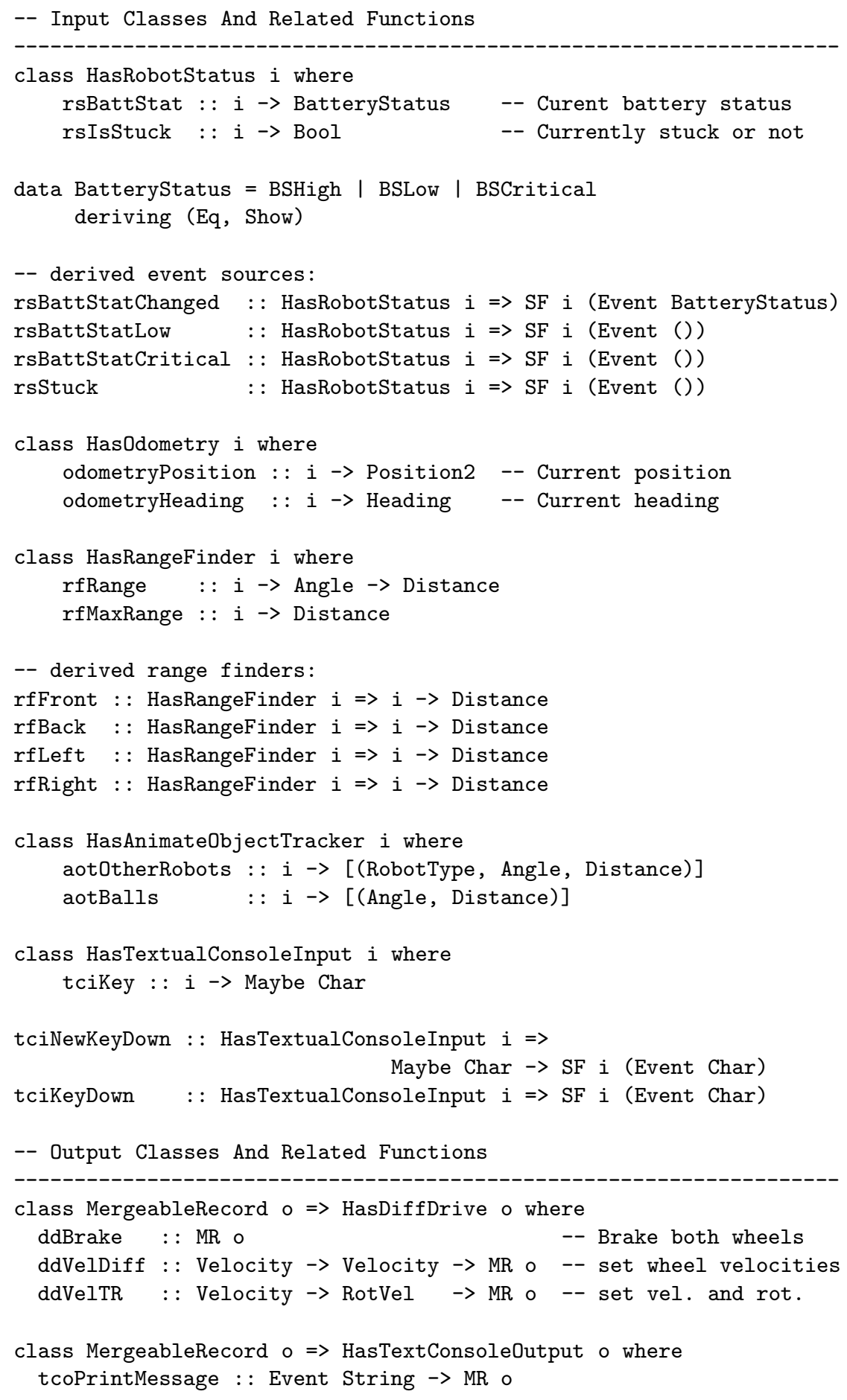

Fig. 3. Robot Input and Output Classes 
For example, the expression:

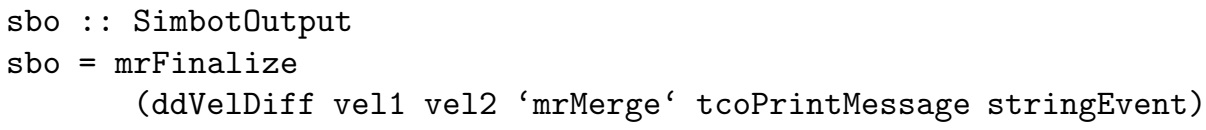

merges the velocity output with a console message.

For simbots, it turns out that velocity control and message output are the only two things that can be merged, so the use of the MergeableRecord class may seem like an overkill. However, for other robots there may be many such mergeable outputs, and the functionality thus offered is quite convenient.

When two common outputs are merged, the result depends on how the mrMerge and mrFinalize methods are defined to behave. The designer of a particular instance of these methods might signal an error, accept one output or the other (for example, merging two calls to ddVelDiff yields the value of the first one), or combine the two (for example, merging two calls to tcoPrintMessage results in both messages being printed in order).

\subsection{Robot Controllers}

To control a robot we must define a robot controller, which, for the case of simbots, must have type:

type SimbotController $=$ SimbotProperties $\rightarrow$ SF SimbotInput SimbotOutput

SimbotProperties is a data type that specifies static properties of a simbot. These properties are accessed abstractly in that SimbotProperties is an instance of the HasRobotProperties type class:

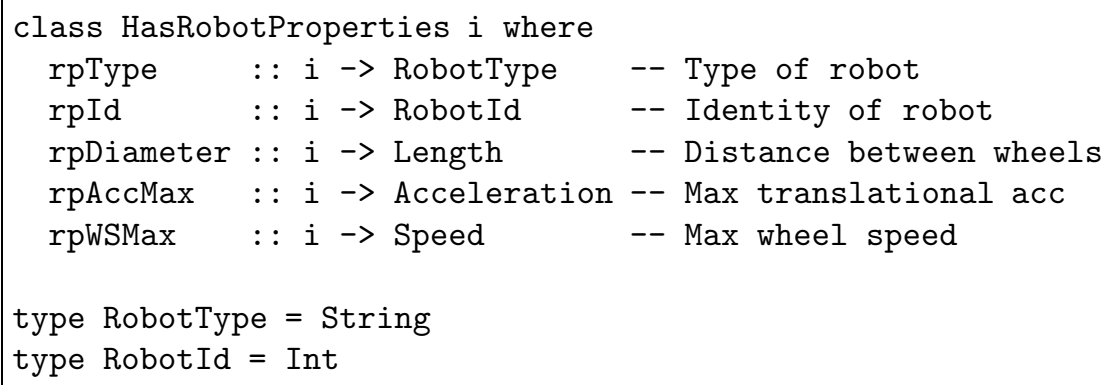

The simulator knows about two versions of the simbot, for which each of these properties is slightly different. The RobotType field is just a string, which for the simbots will be either "SimbotA" or "SimbotB". The remaining fields are self-explanatory.

To actually run the simulator, we use the function:

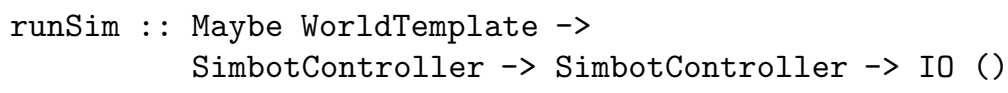


where a WorldTemplate is a data type that describes the initial state of the simulator world. It is a list of simbots, walls, balls, and blocks, along with locations of the centers of each:

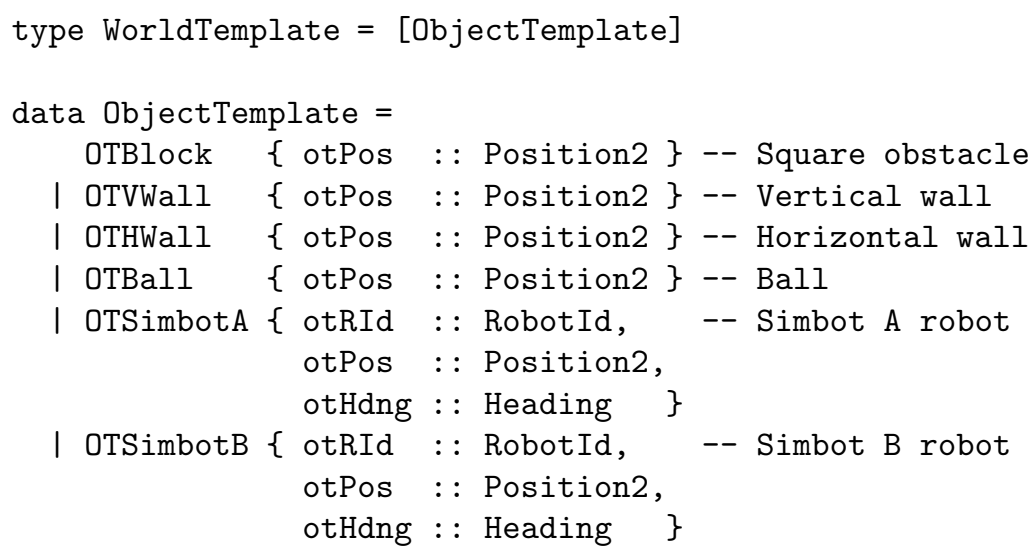

The constants worldXMin, worldYMin, worldXMax, and worldYMax are the bounds of the simulated world, and are assumed to be in meters. Currently these values are $-5,-5,5$, and 5 , respectively (i.e. the world is 10 meters by 10 meters, with the center coordinate being $(0,0)$ ). The walls are currently fixed in size at $1.0 \mathrm{~m}$ by $0.1 \mathrm{~m}$, and the blocks are $0.5 \mathrm{~m}$ by $0.5 \mathrm{~m}$. The diameter of a simbot is $0.5 \mathrm{~m}$.

Your overall program should be structured as follows:

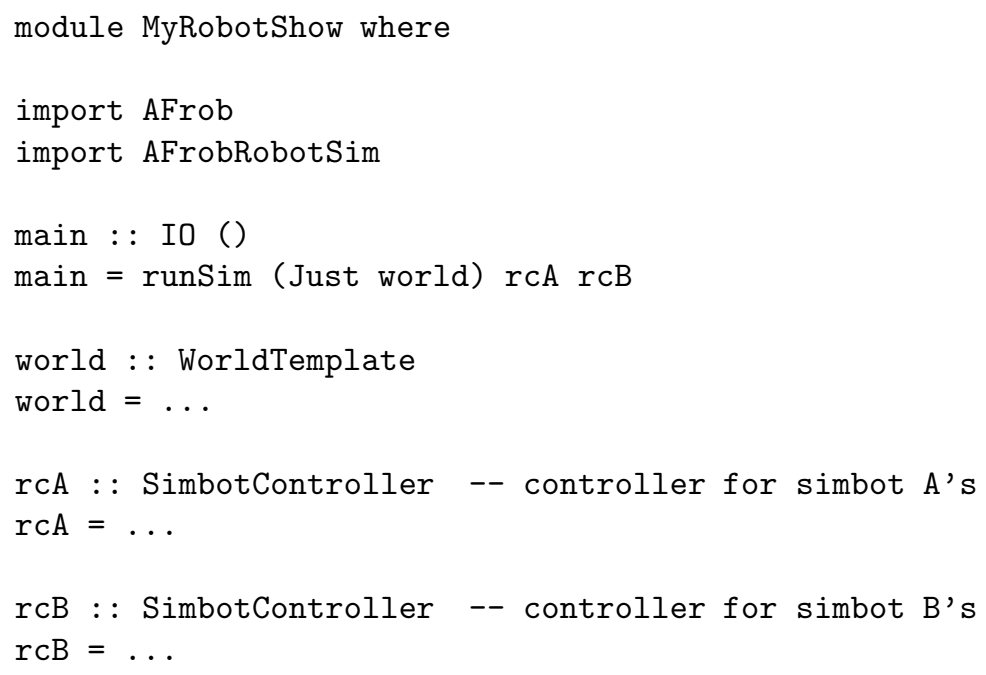


The module AFrob also imports the Yampa library. The module AFrobRobotSim is the robot simulator.

Note that many robots may be created of the same kind (i.e. simbot A or simbot B) in the world template, but the same controller will be invoked for all of them. If you want to distinguish amongst them, simply give them different RobotID's. For example, if you have three simbot A robots, then your code for controller rcA can be structured like this:

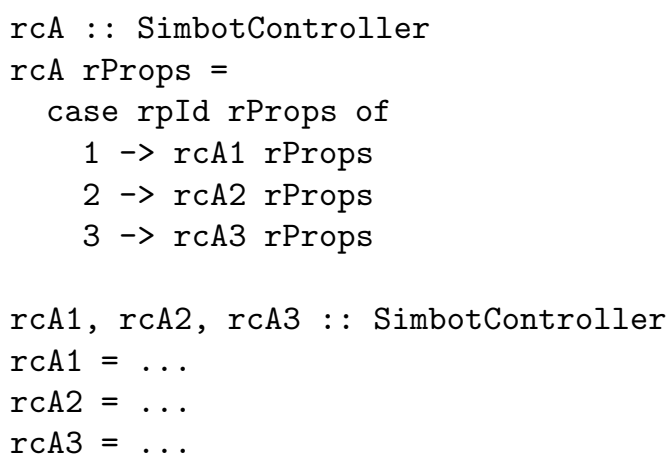

\subsection{Basic Robot Movement}

In this section we will write a series of robot controllers, each of type SimbotController. Designing controllers for real robots is both an art and a science. The science part includes the use of control theory and related mathematical techniques that focus on differential equations to design optimal controllers for specific tasks. We will not spend any time on control theory here, and instead will appeal to the reader's intuition in the design of functional, if not optimal, controllers for mostly simple tasks. For more details on the kinematics of mobile robots, see [3].

Stop, go, and turn. For starters, let's define the world's dumbest controller one for a stationary simbot:

rcStop : : SimbotController

rcStop _ = constant (mrFinalize ddBrake)

Or we could make the simbot move blindly forward at a constant velocity:

rcBlind _ = constant (mrFinalize \$ ddVelDiff 10 10)

We can do one better than this, however, by first determining the maximal allowable wheel speeds and then running the simbot at, say, one-half that speed:

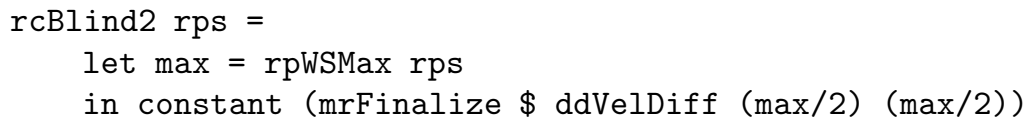


We can also control the simbot through ddVelTR, which allows specifying the simbot's forward and rotational velocities, rather than the individual wheel speeds. For a differential drive robot, the maximal rotational velocity depends on the vehicle's forward velocity; it can rotate most quickly when it is standing still, and cannot rotate at all if it is going at its maximal forward velocity (because to turn while going at its maximal velocity, one of the wheels would have to slow down, in which case it would no longer be going at its maximal velocity). If the maximal wheel velocity is $v_{\max }$, and the forward velocity is $v_{f}$, then it is easy to show that the maximal rotational velocity in radians per second is given by:

$$
\omega_{\max }=\frac{2\left(v_{\max }-v_{f}\right)}{l}
$$

For example, this simbot turns as fast as possible while going at a given speed:

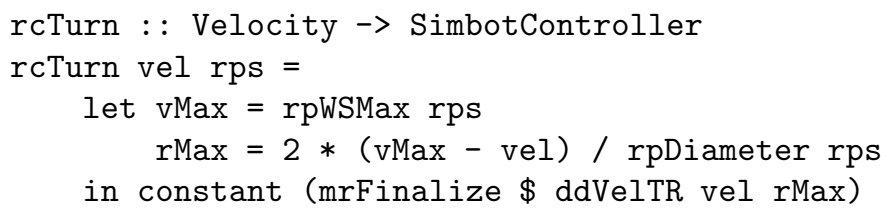

Exercise 7. Link rcBlind2, rcTurn, and rcStop together in the following way: Perform rcBlind2 for 2 seconds, then rcTurn for three seconds, and then do rcStop. (Hint: use after to generate an event after a given time interval.)

The simbot talks (sort of). For something more interesting, let's define a simbot that, whenever it gets stuck, reverses its direction and displays the message "Ouch!!" on the console:

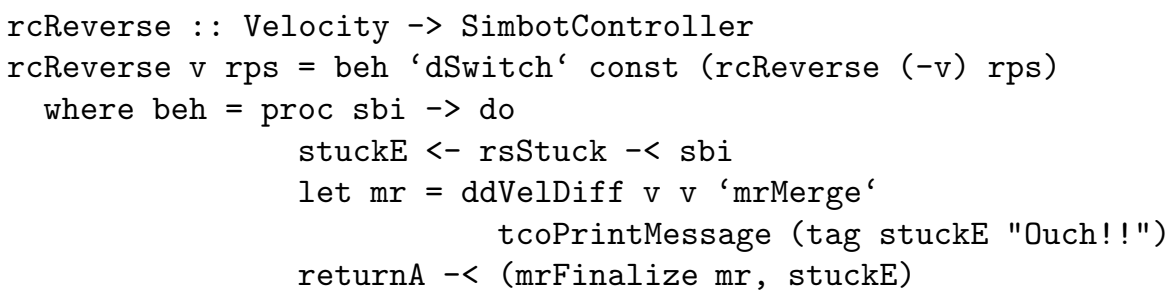

Note the use of a let binding within a proc: this is analogous to a let binding within Haskell's monadic do syntax. Note also that rcReverse is recursive this is how the velocity is reversed everytime the simbot gets stuck - and therefore requires the use of dSwitch to ensure that the recursion is well founded. (It does not require the rec keyword, however, because the recursion occurs outside of the proc expression.) The other reason for the dSwitch is rather subtle: tcoPrintMessage uses stuckE to control when the message is printed, but stuckE also controls the switch; thus if the switch happened instantaneously, the message would be missed! 
If preferred, it is not hard to write rcReverse without the arrow syntax:

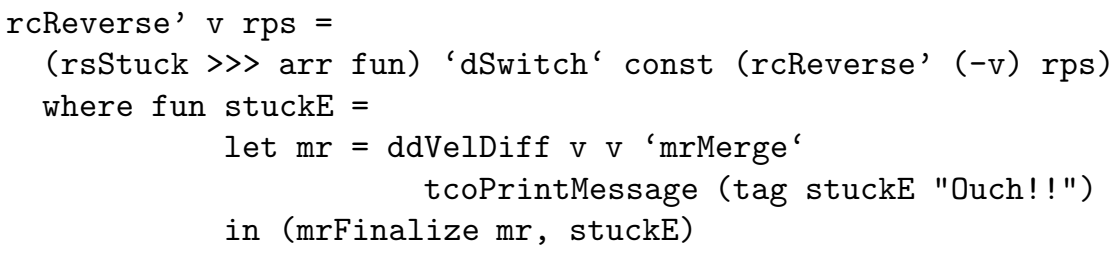

Exercise 8. Write a version of rcReverse that, instead of knowing in advance what its velocity is, takes a "snapshot" of the velocity, as described in Sec. 2.7, at the moment the stuck event happens, and then negates this value to continue.

Finding our way using odometry. Note from Fig. 3 that our simbots have odometry; that is, the ability of a robot to track its own location. This capability on a real robot can be approximated by so-called "dead reckoning," in which the robot monitors its actual wheel velocities and keeps track of its position incrementally. Unfortunately, this is not particularly accurate, because of the errors that arise from wheel slippage, uneven terrain, and so on. A better technique is to use GPS (global positioning system), which uses satellite signals to determine a vehicle's position to within a few feet of accuracy. In our simulator we will assume that the simbot's odometry is perfect.

We can use odometry readings as feedback into a controller to stabilize and increase the accuracy of some desired action. For example, suppose we wish to move the simbot at a fixed speed in a certain direction. We can set the speed easily enough as shown in the examples above, but we cannot directly specify the direction. However, we can read the direction using the odometry function odometryHeading : : SimbotInput -> Heading and use this to control the rotational velocity.

(A note about robot headings. In AFrob there are three data types that relate to headings:

1. Heading is assumed to be in radians, and is aligned with the usual Cartesian coordinate system, with 0 radians corresponding to the positive $\mathrm{x}$-axis, $\pi / 2$ the positive $y$-axis, and so on. Its normalized range is $[-\pi, \pi)$.

2. Bearing is assumed to be in degrees, and is aligned with a conventional compass, with 0 degrees corresponding to north, 90 degrees to east, and so on. Its normalized range is $[0,360)$.

3. Angle is assumed to be in radians, but is a relative measure rather than being aligned with something absolute.

AFrob also provide conversion functions between bearings and headings:

bearingToHeading : : Bearing $\rightarrow$ Heading

headingToBearing : : Heading $\rightarrow$ Bearing

However, in this paper we only use headings and relative angles.) 
Getting back to our problem, if $h_{d}$ and $h_{a}$ are the desired and actual headings in radians, respectively, then the heading error is just $h_{e}=h_{d}-h_{a}$. If $h_{e}$ is positive, then we want to turn the robot in a counter-clockwise direction (i.e. using a positive rotational velocity), and if $h_{e}$ is negative, then we want to turn the robot in a clockwise direction (i.e. using a negative rotational velocity). In other words, the rotational velocity should be directly proportioonal to $h_{e}$ (this strategy is thus called a proportionate controller). One small complication to this scheme is that we need to normalize $h_{d}-h_{a}$ to keep the angle in the range $[-\pi, \pi)$. This is easily achieved using Yampa's normalizeAngle function. Here is the complete controller:

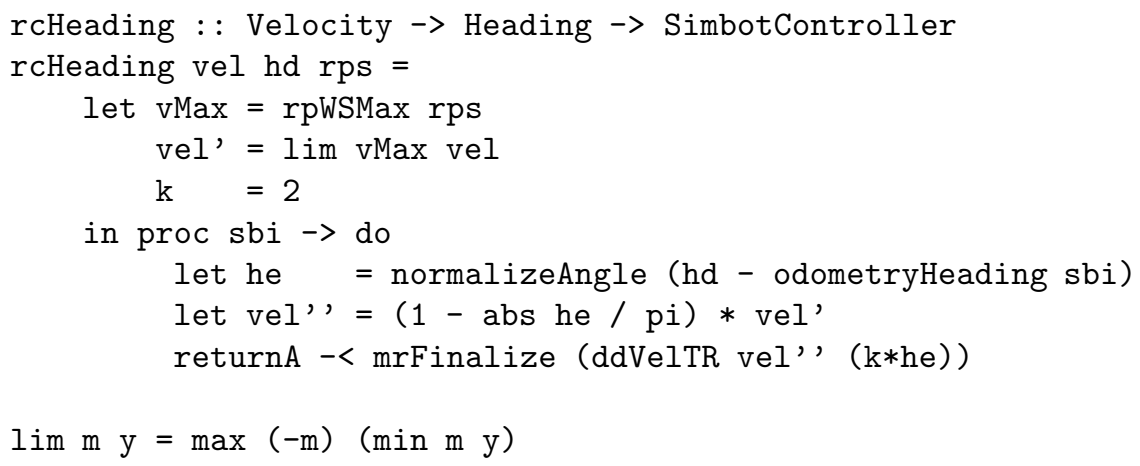

The parameter $k$ is called the gain of the controller, and can be adjusted to give a faster response, at the risk of being too fast and thus being unstable. lim $\mathrm{m} \mathrm{y}$ limits the maximum absolute value of y to $\mathrm{m}$.

Before the next example we will first rewrite the above program in the following way:

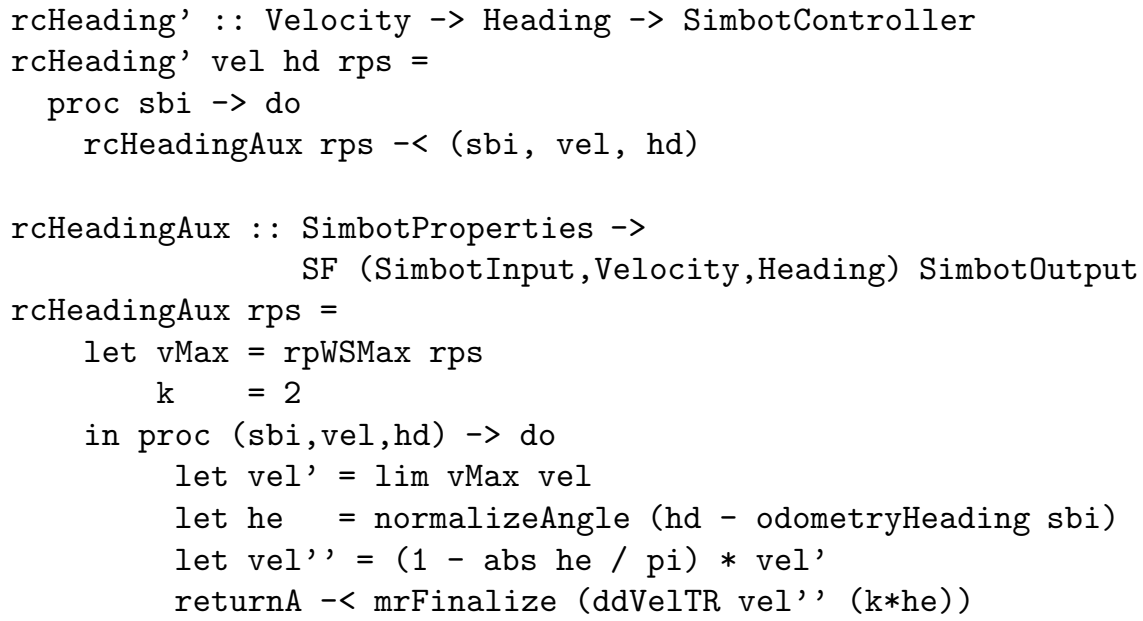

In the original definition, vel and hd were constant during the lifetime of the signal function, whereas in the second version they are treated as signals in 
rcHeadingAux, thus allowing for them to be time varying. Although not needed in this example, we will need this capability below.

As another example of using odometry, consider the task of moving the simbot to a specific location. We can do this by computing a trajectory from our current location to the desired location. By doing this continually, we ensure that drift caused by imperfections in the robot, the floor surface, etc. do not cause appreciable error.

The only complication is that we must take into account our simbot's translational inertia: if we don't, we may overshoot the target. What we'd like to do is slow down as we approach the target (as for rcHeading, this amounts to designing a proportionate controller). Here is the code:

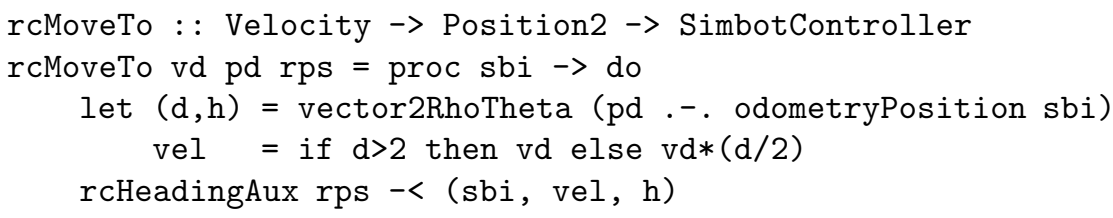

Note the use of vector arithmetic to compute the difference between the desired position pd and actual position odometryPosition sbi, and the use of vector2RhoTheta to convert the error vector into distance $d$ and heading $h$. vel is the speed at which we will approach the target. Finally, note the use of rcHeadingAux defined above to move the simbot at the desired velocity and heading.

Exercise 9. rcMoveTo will behave a little bit funny once the simbot reaches its destination, because a differential drive robot is not able to maneuver well at slow velocities (compare the difficulty of parallel parking a car to the ease of switching lanes at high speed). Modify rcMove so that once it gets reasonably close to its target, it stops (using rcStop).

Exercise 10. Define a controller to cause a robot to follow a sinusoidal path. (Hint: feed a sinusoidal signal into rcHeadingAux.)

Exercise 11. Define a controller that takes a list of points and causes the robot to move to each point successively in turn.

Exercise 12. (a) Define a controller that chases a ball. (Hint: use the aotBalls method in class HasAnimateObjectTracker to find the location of the ball.) (b) Once the ball is hit, the simulator will stop the robot and create an rsStuck event. Therefore, modify your controller so that it restarts the robot whenever it gets stuck, or perhaps backs up first and then restarts.

Home on the range. Recall that our simbots have range finders that are able to determine the distance of the nearest object in a given direction. We will assume that there are four of these, one looking forward, one backward, one to the left, and one to the right: 


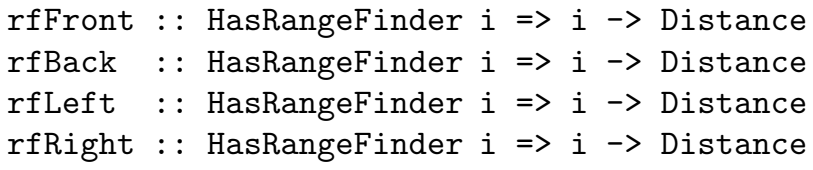

These are intended to simulate four sonar sensors, except that they are far more accurate than a conventional sonar, which has a rather broad signal. They are more similar to the capability of a laser-based range finder.

With a range finder we can do some degree of autonomous navigation in "unknown terrain." That is, navigation in an area where we do not have a precise map. In such situations a certain degree of the navigation must be done based on local features that the robot "sees," such as walls, doors, and other objects.

For example, let's define a controller that causes our simbot to follow a wall that is on its left. The idea is to move forward at a constant velocity $v$, and as the desired distance $d$ from the wall varies from the left range finder reading $r$, adjustments are made to the rotational velocity $\omega$ to keep the simbot in line. This task is not quite as simple as the previous ones, and for reasons that are beyond the scope of this paper, it is desirable to use what is known as a PD (for "proportionate/derivative") controller, which means that the error signal is fed back proportionately and also as its derivative. More precisely, one can show that, for small deviations from the norm:

$$
\omega=K_{p}(r-d)+K_{d}\left(\frac{d r}{d t}\right)
$$

$K_{p}$ and $K_{d}$ are the proportionate gain and derivative gain, respectively. Generally speaking, the higher the gain, the better the reponse will be, but care must be taken to avoid responding too quickly, which may cause over-shooting the mark, or worse, unstable behavior that is oscillatory or that diverges. It can be shown that the optimal relationship between $K_{p}$ and $K_{d}$ is given by:

$$
K_{p}=v K_{d}^{2} / 4
$$

In the code below, we will set $K_{d}$ to 5 . For pragmatic reasons we will also put a limit on the absolute value of $\omega$ using the limiting function lim.

Assuming all of this mathematics is correct, then writing the controller is fairly straightforward:

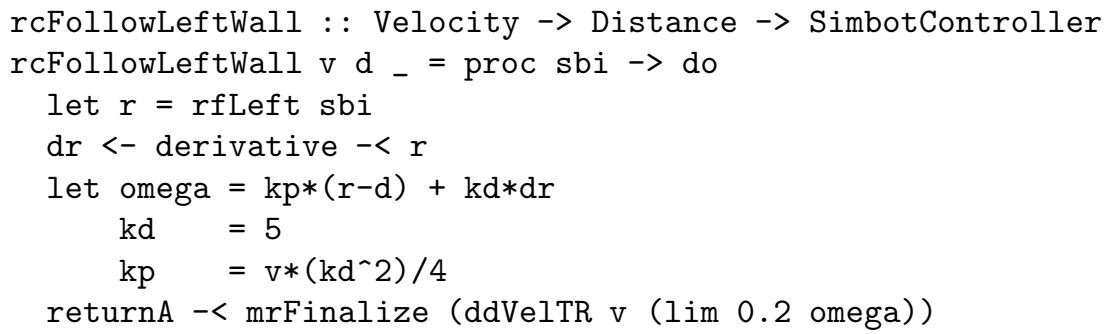


Exercise 13. Enhance the wall-follower controller so that it can make left and right turns in a maze constructed only of horizontal and vertical walls. Specifically:

1. If the simbot sees a wall directly in front of itself, it should slow down as it approaches the wall, stopping at distance $d$ from the wall. Then it should turn right and continue following the wall which should now be on its left. (This is an inside-corner right turn.)

2. If the simbot loses track of the wall on its left, it continues straight ahead for a distance $d$, turns left, goes straight for distance $d$ again, and then follows the wall which should again be on its left. (This is an outside-corner left turn.)

Test your controller in an appropriately designed world template.

Exercise 14. As mentioned in the derivation above, the rcFollowLeftWall controller is only useful once the robot is close to being on track: i.e. at the proper distance from the wall and at the proper heading. If the robot is too far from the wall, it will tend to turn too much in trying to get closer, which makes the left range finder see an even greater distance, and the system becomes unstable. Designing a more robust wall follower is tricky business, and is best treated as multi-mode system, where the robot first seeks a wall, aligns itself parallel to the wall, and then tries to follow it. Design such a controller.

Mass hysteria. As mentioned earlier, the simulator can handle a number of simbots simultaneously. Groups of robots can exhibit all kinds of interesting and productive group behavior (or possibly mass hysteria), limited only by the cleverness of you, the designer. We will describe one simple kind of group behavior here, leaving others (such as the soccer match described in Ex. 16) to you.

The behavior that we will define is that of convergence. Assume that all simbots are initially moving in arbitrary directions and speeds. Each simbot will look at the positions of all of the others, and move toward the centroid (i.e. average) of those positions. If each robot does this continuously and independently, they will all end up converging upon the same point.

To achieve this, recall first the HasAnimateObjectTracker class:

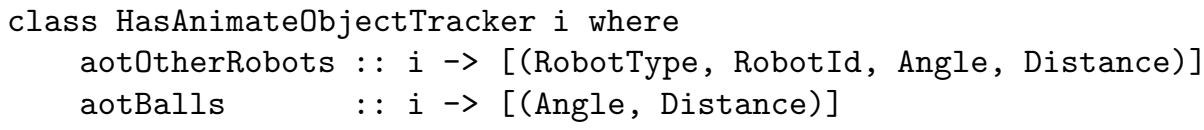

The first of these operations permits us to determine the angle and distance of each of the other simbots. By converting these measurements to vectors, we can add them and take their average, then use rcHeading to steer the robot toward the resulting centroid.

Other than dealing with numeric conversions, the final code is fairly straightforward: 


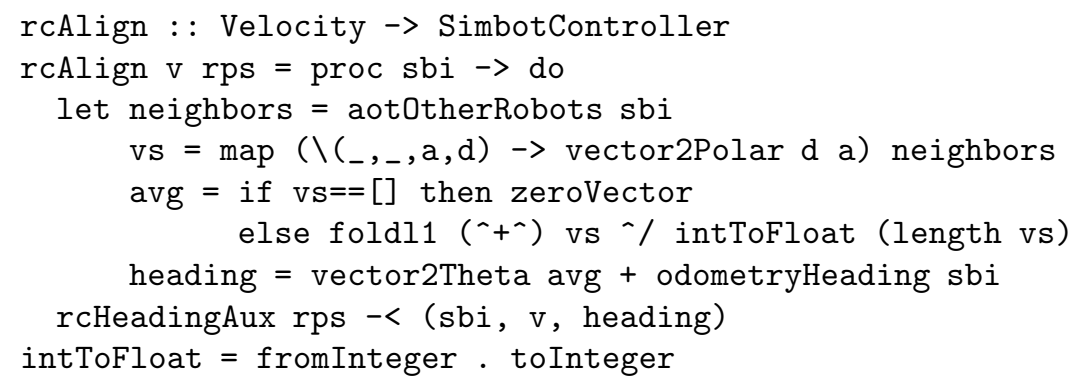

When observing the world through robot sensors, one should not make too many assumptions about what one is going to see, because noise, varying light conditions, occlusion, etc. can destroy those expectations. For example, in the case of the simbots, the simulator does not guarantee that all other robots will be visible through the animate object tracker. Indeed, at the very first time-step, none are visible. For reasons of causality, sensor data is delayed one time-step; but at the very first time step, there is no previous data to report, and thus the animate object tracker returns an empty list of other robots. This is why in the code above the list vs is tested for being empty.

Exercise 15. Write a program for two simbots that are traveling in a straight path, except that their paths continually interleave each other, as in a braid of rope. (Hint: treat the velocities as vectors, and determine the proper equations for two simbots to circle one another while maintaining a specified distance. Then add these velocities to the simbots' forward velocities to yield the desired behavior.)

Exercise 16. Write a program to play "robocup soccer," as follows. Using wall segments, create two goals at either end of the field. Decide on a number of players on each team, and write controllers for each of them. You may wish to write a couple of generic controllers, such as one for a goalkeeper, one for attack, and one for defense. Create an initial world where the ball is at the center mark, and each of the players is positioned strategically while being on-side (with the defensive players also outside of the center circle). Each team may use the same controller, or different ones. Indeed, you can pit your controller-writing skills against those of your friends (but we do not recommend betting money on the game's outcome).

\section{Acknowledgements}

We wish to thank all the members of the Yale Haskell Group for their support and feedback on many of the ideas in this paper. In particular, thanks to Zhanyong Wan, who undoubtedly would have been deeply involved in this work if he had not been so busy writing his thesis. Also thanks to Greg Hager and Izzet Pembeci at Johns Hopkins, who believed enough in our ideas to try them out on real robots. Finally, thanks to Conal Elliott, who started us on our path toward continuous nirvana, despite discrete moments of trauma. 


\section{References}

1. Richard S. Bird. A calculus of functions for program derivation. In David A. Turner, editor, Reseach Topics in Functional Programming. Adison-Wesley, 1990.

2. Antony Courtney and Conal Elliott. Genuinely functional user interfaces. In Proc. of the 2001 Haskell Workshop, September 2001.

3. Gregory Dudek and Michael Jenkin. Computational Principles of Mobile Robots. Cambride University Press, New York, 2000.

4. Conal Elliott. Functional implementations of continuous modeled animation. In Proceedings of PLILP/ALP '98. Springer-Verlag, 1998.

5. Conal Elliott and Paul Hudak. Functional reactive animation. In International Conference on Functional Programming, pages 263-273, June 1997.

6. Paul Hudak. The Haskell School of Expression - Learning Functional Programming through Multimedia. Cambridge University Press, New York, 2000.

7. John Hughes. Generalising monads to arrows. Science of Computer Programming, 37:67-111, May 2000.

8. Henrik Nilsson, Antony Courtney, and John Peterson. Functional Reactive Programming, continued. In ACM SIGPLAN 2002 Haskell Workshop, October 2002.

9. Ross Paterson. A new notation for arrows. In ICFP'01: International Conference on Functional Programming, pages 229-240, Firenze, Italy, 2001.

10. Izzet Pembeci, Henrik Nilsson, and Greogory Hager. Functional reactive robotics: An exercise in principled integration of domain-specific languages. In Principles and Practice of Declarative Programming (PPDP'02), October 2002.

11. John Peterson, Gregory Hager, and Paul Hudak. A language for declarative robotic programming. In International Conference on Robotics and Automation, 1999.

12. John Peterson, Paul Hudak, and Conal Elliott. Lambda in motion: Controlling robots with Haskell. In First International Workshop on Practical Aspects of Declarative Languages. SIGPLAN, Jan 1999.

13. John Peterson, Zhanyong Wan, Paul Hudak, and Henrik Nilsson. Yale FRP User's Manual. Department of Computer Science, Yale University, January 2001. Available at http://www.haskell.org/frp/manual.html.

14. Alastair Reid, John Peterson, Greg Hager, and Paul Hudak. Prototyping realtime vision systems: An experiment in DSL design. In Proc. Int'l Conference on Software Engineering, May 1999.

15. Zhanyong Wan. Functional Reactive Programming for Real-Time Embedded Systems. PhD thesis, Department of Computer Science, Yale University, December 2002.

16. Zhanyong Wan and Paul Hudak. Functional reactive programming from first principles. In Proceedings of the ACM SIGPLAN 'OO Conference on Programming Language Design and Implementation (PLDI), pages 242-252, Vancouver, BC, Canada, June 2000. ACM, ACM Press.

17. Zhanyong Wan, Walid Taha, and Paul Hudak. Real-time FRP. In Proceedings of Sixth ACM SIGPLAN International Conference on Functional Programming, Florence, Italy, September 2001. ACM.

18. Zhanyong Wan, Walid Taha, and Paul Hudak. Event-driven FRP. In Proceedings of Fourth International Symposium on Practical Aspects of Declarative Languages. ACM, Jan 2002. 